\title{
Extraits antigéniques spécifiques obtenus \\ à partir d'Entamoeba bistolytica maintenues en culture mixte
}

\author{
I. Obtention - Caractérisation chimique \\ et immunologique d'un extrait total
}

par J. BENEX

(Collaboration technique Catherine Rouge)

Institut Pasteur, Service de Parasitologie, 25, rue du Docteur-Roux, F. 75 -Paris-15

\section{Résumé}

La saturation par un antigène flore total, d'un immun-sérum anti-amibes + fiore, met en évidence dans toutes les réactions sérologiques la fraction spécifique d'Entamoeba histolytica et ainsi permet d'utiliser dans ces réactions un antigène total, préparé à partir de cultures mixtes E. histolytica + flore, en vue de la mise en évidence des anticorps spécifiques anti-amibes.

L'antigène ainsi obtenu donne des résultats très sensibles et fidèles: en hémagglutination, dans toutes les méthodes de précipitation, diffusion en gélose et immuno-électrophorèse ; sa sensibilité est un peu moindre en réaction de fixation du complément et la particulière difficulté technique de cette réaction nous la fera déconseiller pour un diagnostic pratique.

Par contre, les réactions d'hémagglutination et de précipitation au latex, ainsi que la méthode de double diffusion en gélose peuvent être précieuses comme techniques de référence pour l'étude des sérums immuns.

L'emploi de culture pure n'est donc pas obligatoire pour la préparation d'antigène amibien utilisable en réactions sérologiques. 


\section{Summary}

An antigen extracted from Amoeba Entamoeba histolytica cultivated in vitro with associated intestinal bacteria can be used in serologic tests performed to detect amoeba antibodies.

This antigen react specifically when immune sera were saturated with bacteria antigen prepared in the same way.

Sensibility and specificity are very good in all the serologic tests used: latex agglutination - agar gel diffusion - hemagglutination - immunoelectrophoresis, except in complement fixation test where sensibility is the least.

These techniques could be value as diagnostic aid and epidemiologic investigations.

It seems that associated bacteria have no action on immunogenicity of amoeba strain on the contrary of our leading hypothesis.

La majorité des extraits antigéniques, définis à l'heure actuelle à partir d'Entamoeba histolytica, proviennent de souches maintenues en culture pure (1, 7). Il peut donc paraître paradoxal d'entreprendre un travail sur les antigènes d'E. histolytica en utilisant, comme source de matériel, des amibes maintenues en culture mixte avec la flore bactérienne normalement associée (1).

Il nous a paru cependant intéressant d'aborder ce problème par le fait même que la population d'amibes se trouvait ainsi maintenue dans les conditions se rapprochant le plus de celles rencontrées normalement chez l'hôte parasité.

On sait maintenant d'une façon formelle le rôle indispensable que joue la flore dans la multiplication des trophozoïtes et l'établissement d'une amibiase intestinale.

Ces bactéries, sans lesquelles on ne peut concevoir la présence d'amibes dans l'intestin, sont-elles sans effet sur le potentiel antigénique des souches d'E. histolytica?

Les travaux les plus récents sur l'immunologie des protozoaires font ressortir l'importance des produits métaboliques parmi les antigènes les plus spécifiques. Il nous paraît donc probable que cette flore associée, en contact intime avec le parasite, doit en modifier le métabolisme et par là-même, l'immunogénicité.

Les fractions ainsi obtenues, à partir de souches mixtes, seront-elles plus spécifiques ou tout au moins différentes de celles qu'ont mises en évidence les auteurs travaillant sur des cultures pures? La sensibilité de tels antigènes utilisés dans les différentes réactions sérologiques sera-t-elle, elle aussi, modifiée?

En un mot, la flore bactérienne dans les cultures d'E. histolytica est-elle un avantage ou un inconvénient pour la production d'antigènes spécifiques ?

C'est pour répondre à ces questions que nous avons entrepris ce travail. 


\section{Matériel et Méthode}

Nous avons utilisé une souche d'E. histolytica. Les cultures sont normalement entretenues dans des tubes de $17 \mathrm{~mm}$ et repiquées tous les $4 / 5$ jours, comme l'a décrit L. Lamy en 1964 (6).

La préparation d'antigènes nécessitant une quantité importante de protozoaires, les amibes sont ensemencées sur des erlenmeyers de $100 \mathrm{ml}$. Après 5 jours, la culture est suffisamment riche pour permettre une récolte valable. Chaque préparation antigénique est faite à partir du contenu de 2 erlenmeyers de $100 \mathrm{ml}$.

\section{Méthode de préparation de l'extrait antigénique.}

Les erlenmeyers sont sortis de l'étuve à $37^{\circ} \mathrm{C}$ et rapidement refroidis dans un bain de glace pendant 10 minutes. Ceci a pour effet de détacher les amibes des parois et de les faire toutes se rassembler au fond de l'erlenmeyer, sans les tuer.

Le contenu des 2 erlenmeyers est alors rassemblé et centrifugé pendant 10 minutes à $500 \mathrm{G}$.

Le culot obtenu qui correspond aux amibes, à la flore associée et à l'amidon, est lavé trois fois dans une solution de saccharose à $0,25 \mathrm{M}$ qui évite la lyse des trophozoïtes. Ces centrifugations et lavages permettent d'éliminer une partie de la flore microbienne. Le sédiment est alors repris dans du tampon phosphate à $\mathrm{pH}=7,2$, puis soumis à quatre congélations et décongélations successives. Le nombre de quatre a été choisi, après expérimentation, comme étant suffisant pour faire éclater la totalité des trophozoïtes d'amibes, mais demeure insuffisant pour désintégrer les bactéries.

Au cours de ces manipulations successives, nous sommes ainsi arrivés à éliminer la plus grande partie des bactéries, ne conservant que des amibes partiellement éclatées et l'amidon.

A partir de ce stade, l'extraction proprement dite des composants antigéniques va intervenir. Deux procédés différents ont été expérimentés :

a) Passage au broyeur pendant 10 minutes à 200 tours $/ \mathrm{mn}$, puis extraction sur agitateur magnétique une nuit à $+4^{\circ} \mathrm{C}$.

b) Passage aux ultra-sons à l'aide d'un appareil Piezo-Ceram électronique. Pour la désintégration des protozoaires, la fréquence du bain $\mathrm{n}^{\circ} 2$ correspond à $500 \mathrm{k}$ hertz avec une puissance de $150 \mathrm{~mA}$, ceci pendant 5 heures.

Enfin, une ultime centrifugation à $+4^{\circ} \mathrm{C}$ pendant 15 minutes à 8.000 tours $/ \mathrm{mn}$ permet de recueillir un surnageant qui constitue l'antigène brut. 


\section{Etude chimique de l'extrait antigénique brut.}

Afin de permettre une standardisation des différents lots d'antigènes préparés, nous avons choisi une détermination chimique du contenu en protéines des extraits, de préférence à la numération des trophozoïtes. Cette caractérisation nous appparaît meilleure pour refléter les possibilités immunologiques des diverses préparations, les numérations étant faussées par l'existence des conglomérats.

Les protéines sont ainsi déterminées par la méthode spectrocolorimétrique de Lowry, à une longueur d'onde de $280 / \mathrm{m} \mu$.

\section{Résultats.}

TENEUR EN PROTÉINES DES LOTS D'ANTIGÈNE AMIBIEN BRUT $(\mathrm{mg} / \mathrm{ml})$.

\begin{tabular}{|c|c|c|c|c|c|}
\hline \multirow[b]{3}{*}{$\mathrm{A}_{1}$} & \multirow[b]{3}{*}{$\ldots \ldots$} & \multicolumn{2}{|c|}{ Extraction mécanique } & \multicolumn{2}{|c|}{ Ultra-sons } \\
\hline & & $10 \mathrm{mn}+1$ nuit & $1 \mathrm{~h}+1$ nuit & $1 \mathrm{~h}$ & $5 \mathrm{~h}$ \\
\hline & & 0,33 & 0,37 & 0,40 & 0,70 \\
\hline$A_{2}$ & $\cdots \cdots$ & 0,67 & 0,65 & 1,10 & 1,35 \\
\hline
\end{tabular}

ETUdE IMMUNOLOGIQUE.

Réactions utilisées: nous avons utilisé, pour évaluer la valeur immunologique de nos antigènes, six réactions sérologiques $=$ réaction de fixation du complément, test au latex, hémagglutination, double diffusion en gélose, cuves de Oudin, immunoélectrophorèse.

La R.F.C. a été pratiquée selon la technique décrite par Fulton, Joyner et Orpwood Price (3). Cette méthode, mise au point spécialement pour la recherche des anticorps amibiens, présente des modalités particulières. Pour les détails techniques, nous renvoyons à la publication des auteurs précités.

Les réactions d'hémagglutination ont été faites selon la technique décrik; par Kessel (4, 5).

Sérums: les sérums utilisés dans ces réactions sont, pour certains, des sérums de malades atteints d'amibiase hépatique ou intestinale aiguë, mais la plupart sont des sérums de lapins hyperimmunisés.

Hyperimmunisation des lapins: chaque lapin reçoit au total $25 \mathrm{mg}$ de protéines en cinq injections de $1 \mathrm{ml}$ tous les cinq jours selon le schéma suivant:

$1^{\text {re }}$ inj. intradermique $+50 \%$ adjuvant de Freund $=6 \mathrm{mg}$

$2^{\circ}$ inj. intramusculaire $\quad=6 \mathrm{mg}$

$3^{\circ}$ inj. intraveineuse $=3 \mathrm{mg}$

$4^{\circ}$ inj. intramusculaire $\quad=7 \mathrm{mg}$

$5^{\circ}$ inj. intraveineuse $\quad=3 \mathrm{mg}$

Saignée 8 jours après la dernière injection. 
Deux séries d'animaux sont ainsi préparées; les uns avec un antigène total = amibe + flore associée, les autres avec un antigène flore, dépourvu d'amibes.

Lors de leur utilisation, les sérums obtenus à partir du premier lot d'animaux hyperimmunisés à l'aide d'un antigène amibe + flore sont saturés par l'antigène flore totale, 1 heure à $37^{\circ} \mathrm{C}$, afin de ne conserver que les anticorps spécifiques des amibes.

Les sérums saturés sont ramenés par concentration sur Carbovax à leur concentration initiale. sérums.

Les résultats dans les différentes réactions sont donnés pour les trois types de

\section{Réactivité de l'Antigène amibien brut dans les réactions sérologiques de référence}

\section{1) Test d'agglutination au latex.} brut.

Les particules de latex (Difco $81 \mu$ ) sont sensibilisées par l'antigène amibien

Un titrage préalable permet de déterminer les proportions optimales à utiliser.

Antigène amibien $A_{2}=0,67 \mathrm{mg} / \mathrm{ml}$ protéines.

Les résultats sont donnés dans le tableau suivant:

Titrage DE L'ANTIGÈNe

Latex Difco $0,81 \mu$

Antigène amibien $0,67 \mathrm{mg} / \mathrm{ml}$ protéines

\begin{tabular}{|c|c|c|c|}
\hline Antigène & Sérum & Sérum négatif & Sérum positif \\
\hline $\begin{array}{l}2 \text { Volume } \\
2 \mathrm{~V}\end{array}$ & $\begin{array}{l}\mathrm{Ag} \\
\text { Latex }\end{array}$ & agglutination 0 & agglutination + \\
\hline $\begin{array}{ll}1 \mathrm{~V} \\
2 \mathrm{~V}\end{array}$ & $\begin{array}{l}\mathrm{Ag} \\
\mathrm{L}\end{array}$ & agglutination 0 & agglutination 0 \\
\hline $\begin{array}{ll}2 & \mathrm{~V} \\
1 \mathrm{~V} \\
\end{array}$ & $\begin{array}{l}\mathrm{Ag} \\
\mathrm{L}\end{array}$ & agglutination 0 & agglutination ++++ \\
\hline $\begin{array}{ll}1 & \mathrm{~V} \\
3 & \mathrm{~V}\end{array}$ & $\begin{array}{l}\mathrm{Ag} \\
\mathrm{L}\end{array}$ & agglutination 0 & agglutination 0 \\
\hline $\begin{array}{l}3 \mathrm{~V} \\
1 \mathrm{~V}\end{array}$ & ${ }_{\mathrm{L}}^{\mathrm{Ag}}$ & légère agglutination & agglutination ++++ \\
\hline
\end{tabular}

Réactions: on choisit alors comme taux de sensibilisation $=2 \mathrm{vol}$. Ag. titrant $0,67 \mathrm{mg} / \mathrm{ml}$ de protéines pour 1 volume de latex $0,81 \mu$. 
Un tel antigène se conserve plusieurs mois à une température de $+10^{\circ} \mathrm{C}$. Il permet de tester des sérums suspects dans des réactions quantitatives.

\begin{tabular}{|c|c|c|c|c|c|c|c|c|c|c|}
\hline Sérum Dilution & pur & $1 / 2$ & $1 / 4$ & $1 / 8$ & $1 / 16$ & $1 / 32$ & $1 / 64$ & $1 / 128$ & $1 / 256$ & $1 / 512$ \\
\hline $\begin{array}{l}\text { sérum amibien } \\
\text { brut } \ldots \ldots \ldots \ldots\end{array}$ & $+++t$ & +++ & +++ & +++ & +++ & ++ & ++ & + & \pm & 0 \\
\hline $\begin{array}{l}\text { sérum amibien } \\
\text { saturé } \mathrm{Ag} \text {. flore }\end{array}$ & +++ & +++ & +++ & +++ & ++ & + & \pm & & & \\
\hline sérum antiflore & ++ & ++ & + & 一 & & & & & & \\
\hline sérum négatif. & 一 & - & 一 & 一 & & & & & & \\
\hline
\end{tabular}

\section{2) Réaction de fixation du complément.}

Les résultats seront présentés sous forme de tableau.

\section{ANTIGÈNE AMIBIEN}

\begin{tabular}{|l|c|c|c|c|c|c|c|c|c|c|}
\hline Sérum Dilution & $1 / 5$ & $1 / 10$ & $1 / 20$ & $1 / 30$ & $1 / 40$ & $1 / 80$ & $1 / 160$ & $1 / 320$ & $1 / 640$ & $1 / 1280$ \\
\hline $\begin{array}{l}\text { sérum anti- } \\
\text { amibes + flore }\end{array}$ & + & + & + & + & + & + & + & + & + & - \\
\hline \begin{tabular}{l} 
sérum antiflore \\
\hline $\begin{array}{l}\text { sérum anti- } \\
\text { amibes saturé } \\
\text { flore }\end{array}$
\end{tabular} & ++ & + & + & + & + & + & + & - & - &.- \\
\hline
\end{tabular}

En contre-épreuve, les mêmes sérums sont testés envers un antigène flore.

\section{ANTIGÈNE FLORE}

\begin{tabular}{|c|c|c|c|c|c|c|c|c|c|c|c|c|c|}
\hline Sérum & pur & $1 / 2$ & $1 / 4$ & $1 / 8$ & $1 / 16$ & $1 / 32$ & $1 / 64$ & $1 / 128$ & $1 / 256$ & $1 / 512$ & $1 / 1024$ & $1 / 2048$ & $1 / 4896$ \\
\hline $\begin{array}{l}\text { sérum } \\
\text { antiflore .. }\end{array}$ & + & + & + & + & + & + & + & $\because=$ & (干) & - & 一 & 一 & - \\
\hline $\begin{array}{l}\text { sérum ami- } \\
\text { bes + flore }\end{array}$ & + & + & + & + & + & + & + & + & + & + & $\%$ & $\overline{7}$ & - \\
\hline $\begin{array}{l}\text { sérum } \\
\text { anti-amibes } \\
\text { saturé flore }\end{array}$ & $\mp$ & - & - & - & - & - & - & - & - & - & - & - & - \\
\hline
\end{tabular}




\section{3) Hémagglutination.}

Les mêmes épreuves sont exécutées par la technique d'hémagglutination (acide tannique).

Les résultats sont résumés dans les deux tableaux suivants:

\section{ANTIGÈNE AMIBIEN}

\begin{tabular}{|c|c|c|c|c|c|c|c|c|}
\hline $\begin{array}{l} \\
\text { Sérum }\end{array}$ & $1 / 8$ & $1 / 32$ & $1 / 128$ & $1 / 512$ & $1 / 2048$ & $1 / 8192$ & $1 / 32768$ & $1 / 131072$ \\
\hline $\begin{array}{l}\text { sérum anti- } \\
\text { amibes }+ \text { flo- } \\
\text { re } \ldots \ldots \ldots \ldots\end{array}$ & + & + & + & + & \pm & - & - & - \\
\hline $\begin{array}{l}\text { sérum anti- } \\
\text { flore } \ldots \ldots \ldots\end{array}$ & + & + & + & + & + & + & \pm & - \\
\hline $\begin{array}{l}\text { sérum anti- } \\
\text { amibes saturé } \\
\text { flore } \quad \ldots \ldots \ldots \text {. }\end{array}$ & + & + & + & + & - & - & - & - \\
\hline
\end{tabular}

ANTIGÈNE FLORE

\begin{tabular}{|c|c|c|c|c|c|c|c|c|}
\hline$\overbrace{\text { Sérum }}^{\text {Dilution }}$ & $1 / 8$ & $1 / 32$ & $1 / 128$ & $1 / 512$ & $1 / 2048$ & $1 / 8192$ & $1 / 32768$ & $1 / 131072$ \\
\hline $\begin{array}{l}\text { sérum anti- } \\
\text { amibes }+ \\
\text { flore } \ldots \ldots .\end{array}$ & +++ & ++ & ++ & ++ & + & + & - & 一 \\
\hline $\begin{array}{l}\text { sérum anti- } \\
\text { flore } \ldots . . .\end{array}$ & +++ & +++ & +++ & +++ & +++ & + & \pm & 一 \\
\hline $\begin{array}{ll}\text { sérum } & \text { anti- } \\
\text { amibes } & \text { satu- } \\
\text { ré flore } & . . .\end{array}$ & - & - & - & - & - & 一 & - & 一 \\
\hline
\end{tabular}

La lecture de ces tableaux met en évidence:

a) L'efficacité de la saturation des sérums totaux (amibes + flore) par l'antigène flore ; seuls, les anticorps anti-Entamoeba histolytica demeurent présents et donnent une réaction spécifique jusqu'au $1 / 80$ en réaction de fixation du complément et jusqu'au $1 / 512$ en hémagglutination.

b) Un double phénomène au niveau des sérums totaux anti-amibes + flore :

- d'une part, les anticorps anti-amibes sont masqués par les anticorps anti-flore et apparaissent après saturation : 
- d'autre part, une partie des anticorps anti-flore est inhibée par la présence des anticorps anti-amibes puisque les taux obtenus sont plus élevés lorsqu'on met en présence un sérum anti-flore pur et un antigène flore sans amibes.

c) La concordance des résultats obtenus en réaction de fixation du complément et en hémagglutination, cette dernière réaction se révélant seulement plus sensible.

\section{4) Diffusion en gélose.}

Trois techniques sont utilisées pour étudier les lignes de précipitation obtenues avec l'antigène amibien : méthode d'Ouchterlony en boîtes de Petri, cuves de Oudin et immunoélectrophorèse.

$1^{\circ}$ Méthode D'Ouchterlony: l'antigène est utilisé à une concentration de $1,11 \mathrm{mg} / \mathrm{ml}$ protéines; les godets sont distants de $5 \mathrm{~mm}$ du godet central.

Dans le cas de la Fig. $\mathrm{n}^{\circ}$ 1, l'antigène amibien total est au centre de la boîte de Petri. Dans les godets périphériques sont disposés en 1 et 2 des sérums anti-amibes bruts, en 3 un sérum anti-flore, en 4 un sérum amibien saturé flore et en 5 un sérum négatif.

Quatre lignes de précipitation apparaissent entre l'antigène et le godet contenant les sérums totaux, 3 bandes correspondent à la flore (godet $\mathrm{n}^{\circ} 3=$ sérum anti-flore), une est spécifique de l'amibe (godet $\mathrm{n}^{\circ} 4$ ).

La Fig. $n^{\circ} 2$ représente la contre-épreuve et l'analyse du milieu de culture.

Au centre, on place un sérum anti-amibes total. A la périphérie, on dispose :

- en 1) l'antigène flore,

- en 2) l'antigène amibes + flore,

- en 3) la phase liquide du milieu de culture avant ensemencement,

- en 4) le même milieu après 5 jours de culture,

— en 5) la phase figurée du milieu de culture.

On obtient encore quatre traits de précipitation entre l'antigène amibien total et le sérum anti-amibes et trois traits contre le sérum anti-flore. Les composants du milieu avant ensemencement ne présentent aucune réaction; mais, au contraire, la phase liquide du milieu après cinq jours de culture laisse apparaître un trait de précipitation avec le sérum amibien total. Ceite réaction est sans doute due à la présence dans le milieu des produits de métabolisme.

Des recherches ultérieures portant sur l'étude de cet antigène devraient apporter des données intéressantes.

$2^{\circ}$ Cuves de Oudin : des résultats comparables sont obtenus en cuves de Oudin.

$3^{\circ}$ ImMUNO-Électrophorèse: l'analyse immuno-électrophorétique des antigènes contre le sérum anti-amibes + flore révèle cinq arcs. Après saturation, le sérum ne contenant plus que les anticorps amibiens révèle encore trois arcs spécifiques d'Entamoeba histolytica: un majeur, deux mineurs (Fig. $\mathrm{n}^{\circ} 3$ ). 


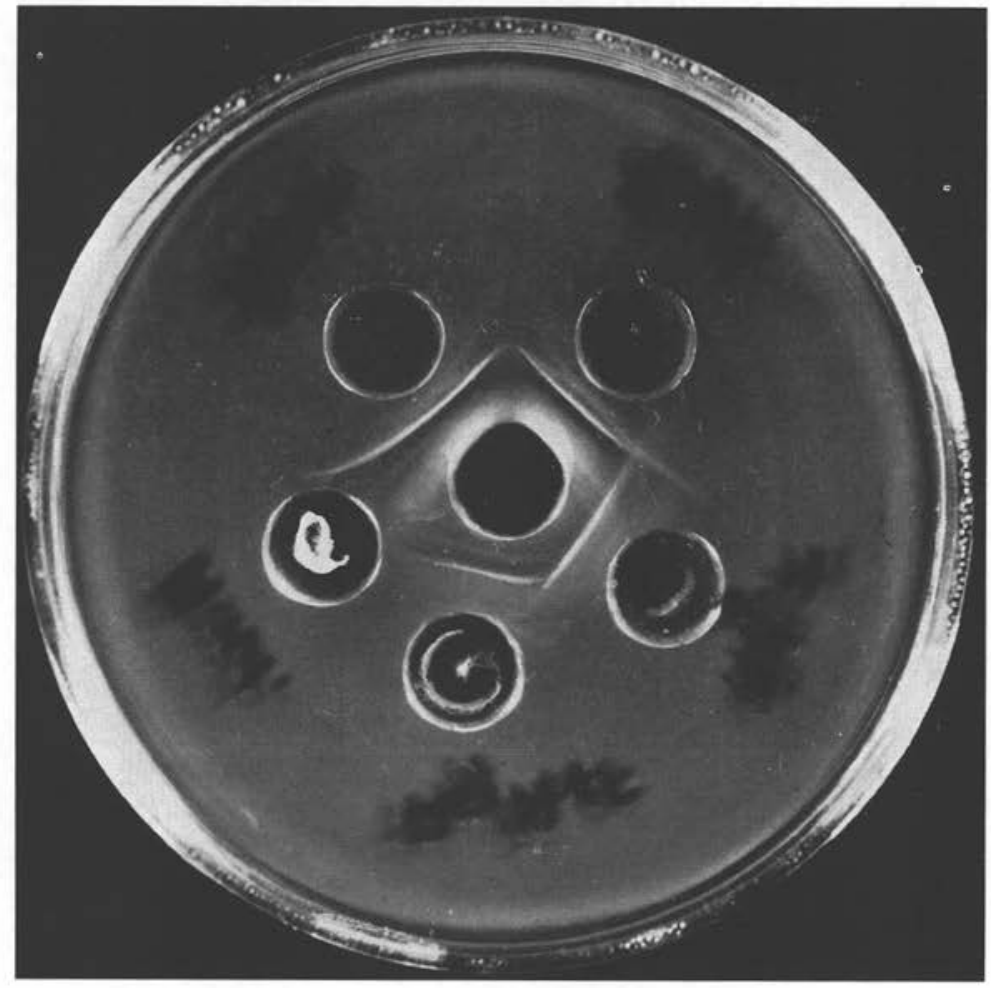

FIG. ${ }^{0} 1$

Au centre: antigène amibien total + flore.

A la périphérie: 1 . sérum amibien total, en haut à gauche; 2 . sérum amibien total, en haut à droite ; 3. sérum anti-flore, en bas à droite: 4. sérum amibien saturé flore en bas; 5 . sérum négatif, à gauche. 


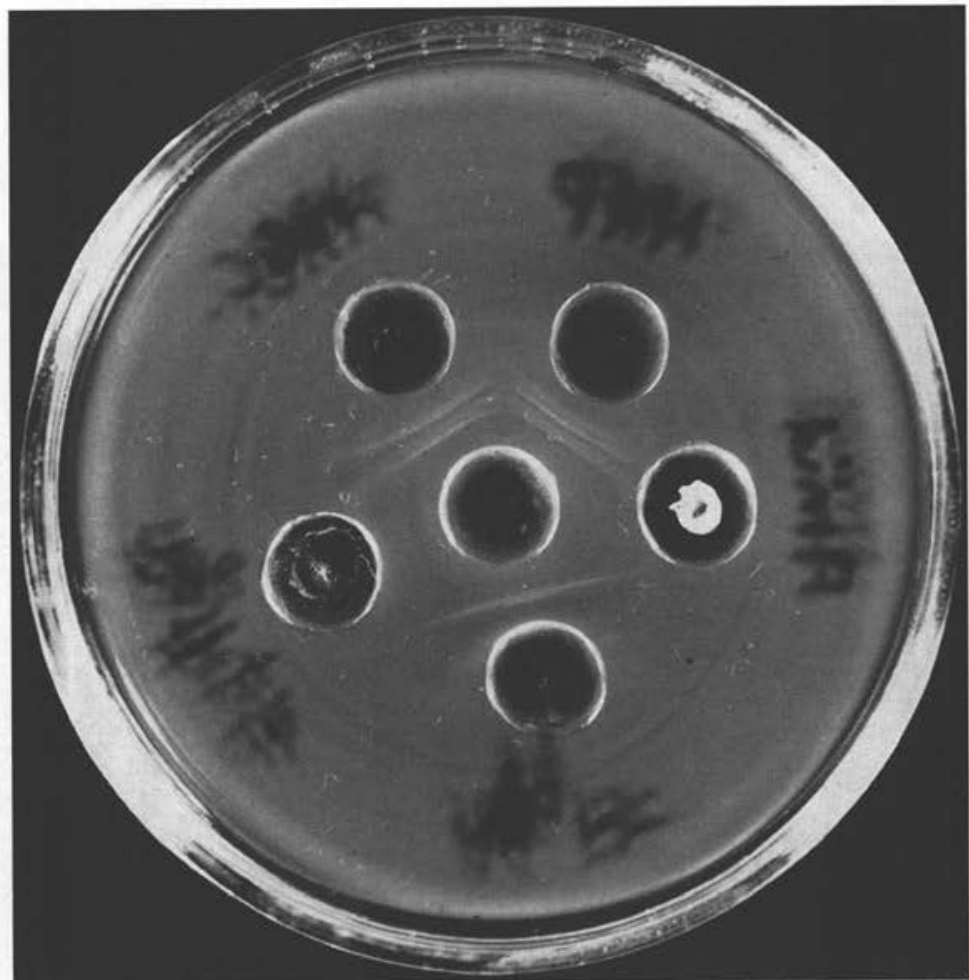

FIG. $N^{\circ} 2$

Au centre: sérum amibien total.

A la périphérie: 1 . antigène flore, en haut à gauche; 2 . antigène amibes + flore, en haut à droite; 3 . phase liquide du milieu de culture à droite ; 4 . idem. après 5 jours de culture en bas; 5 . phase solide du milieu de culture, à gauche.

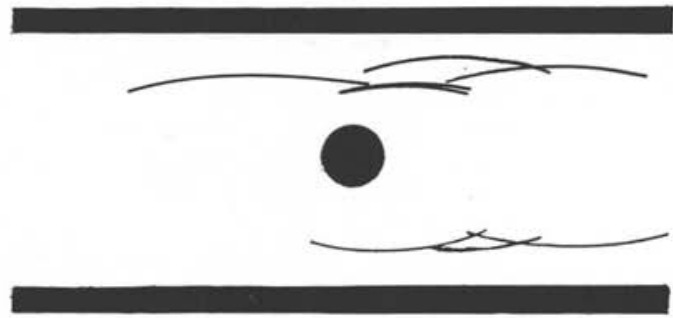

FIG. $\mathrm{N}^{\circ} 3$

Immuno-électrophorèse

Au centre: antigène amibien.

En haut: sérum amibien total.

En bas: sérum amibien saturé flore. 
Etude comparative avec les résultats obtenus à partir de culture pure d'Entamoeba histolytica.

Nous avons pris comme référence les résultats publiés par P.-E. Thomson et coll., en 1968 (8).

Les auteurs utilisent des souches maintenues en culture monophasique bactériologiquement stérile, contenant un milieu complexe, comprenant de l'extrait de foie, de la trypticase, du sérum de cheval, de la cystéine et des vitamines.

Ces auteurs ainsi que d'autres (Schneider et coll., 1968) rapportent que la composition du milieu de culture n'influe pas sur le potentiel antigénique de l'amibe.

Notre propre milieu de culture étant relativement moins complexe que les milieux décrits (bien que comprenant pour une part des constituants similaires), les divergences possibles ne pourront provenir que de la flore bactérienne associée ou d'un métabolisme différent des amibes.

ANTIGÈNE BRUT TOTAL

Taux maximal de positivité des sérums

\begin{tabular}{|c|c|c|}
\hline & culture mixte & culture pure \\
\hline R.F.C. $\ldots \ldots \ldots \ldots \ldots$ & $+1 / 80$ & $1 / 128$ \\
\hline Diffusion en gélose .. & $\begin{array}{l}1 \text { arc en D.D.G. } \\
3 \text { arcs en immuno-électropho- } \\
\text { rèse }\end{array}$ & 3 arcs dont 2 majeurs \\
\hline H.A. $\quad \ldots \ldots \ldots \ldots \ldots$ & $1 / 8192$ & $1 / 10240$ \\
\hline
\end{tabular}

Les résultats obtenus avec les antigènes provenant des deux souches d'amibes paraissent superposables, en ce qui concerne l'antigène brut total avec cependant une sensibilité légèrement inférieure en R.F.C. pour l'antigène brut (1/80 au lieu de 1/128) et en hémagglutination (1/8192 pour 1/10240).

En ce qui concerne les techniques de diffusion en gélose, les comparaisons sont plus difficiles. Il serait nécessaire de comparer les deux antigènes au cours de la même expérimentation.

\section{Conclusion}

Il apparaît donc que, contrairement, à notre hypothèse du départ, la flore bactérienne associée joue un rôle négligeable dans les propriétés immunologiques des amibes Entamoeba histolytica.

Ce travail a, par contre, fait apparaître l'existence d'un antigène pouvant provenir des produits de métabolisme des amibes, antigène que l'on retrouve dans le milieu de culture et dont l'étude devrait conduire à des résultats intéressants. 
Enfin, il se confirme que l'on peut extraire à partir de cultures d'amibes, tant de culture pure que de culture mixte, des antigènes sensibles et spécifiques qui peuvent être utilisés dans les divers tests sérologiques classiques.

L'emploi de culures pures n'est pas essentiel pour ce faire ; néanmoins, leur utilisation évite une manipulation longue et fastidieuse: la préparation d'un antigène à partir de la flore associée et la saturation par cet antigène des sérums à tester.

\section{Bibliographie}

(1) Ali Khan (Z.) et Meerovitch (E.), 1968. - A comparative study of the antigens of some of the « histologita type » strains of Entamoeba. A qualitative and quantitative evaluation of antigens by indirect hemagglutination, gel de précipitation et immunoélestrophorèse. Amer. J. Trop. Med. Hyg., 17, 528-39.

(2) BéNEX (J.), 1970. - Obtention d'un extrait antigénique spécifique à partir d'Entamoeba histolytica maintenues en culture mixte. C.R. Acad. Sc. Paris, 270, 970-72.

(3) Fulton (J. D.), Joyner (L. P.) and OrPwood Price, 1951. - Studies on protozoa. IV. A complement fixation test for amoebiasis. Amer. J. Trop. Med. Hyg., 54, 27-33.

(4) Kessel (J.-P.), Lewis (W. P.), Molina Posquel (C.) and Turner (J. A.), 1965. - Indirect hemagglutination and complement fixation test in amebiasis. Amer. J. Trop. Med. Hyg., 14, 540-550.

(5) Kessel (J.-P.), Lewis (W. P.), Solomon (M. A.) and KiM (M.), 1961. - Preliminary report on a hemagglutination test for Entamoeba. Proc. Soc. Exp. Biol. Med., 106, 409-13.

(6) LAMY (L.) et PiÉchaud (D.), 1963. - Utilisation de la sensibilité bactérienne aux antibiotiques pour l'étude du rôle des bactéries dans le déterminisme de l'enkystement spontané d'Entamoeba histolytica en culture. Ann. Inst. Pasteur, 104, 141-45.

(7) Maddison (S. E.), Kagan (I. G.) and Elsdon-Dew (R.), 1968. - Comparison of intradermal and serologic tests for the diagnosis of amebiasis. Amer. J. Trop. Med. Hyg., 17, 540-47.

(8) Thompson (P. E.), Graedel (S. K.), Schneider (C. R.), Stucki (W. P.) et Gordon (R. M.), 1968. - Preparation of standardized amoeba antigen from axenic cultures of Entamoeba histolytica. Bull. O.M.S., 39, 349-65. 